\title{
Rectus Sheath Hematoma: A Simplified Emergency Surgical Approach
}

\author{
Michael Mantelas ${ }^{1, *}$, Niki Katsiki ${ }^{2,3}$, Polychronis Antonitsis ${ }^{1}$, Ellada Kyurdzhieva ${ }^{4}$, \\ Dimitri P. Mikhailidis ${ }^{2}$ and Apostolos Hatzitolios ${ }^{3}$ \\ ${ }^{I}$ Department of Cardiothoracic Surgery, AHEPA University Hospital, Thessaloniki, Greece \\ ${ }^{2}$ Department of Clinical Biochemistry (Vascular Disease Prevention Clinics), Royal Free Hospital campus, University \\ College London Medical School, University College London (UCL), London NW3 2QG, UK \\ ${ }^{3} 1$ st Propedeutic Department of Internal Medicine, AHEPA University Hospital, Thessaloniki, Greece \\ ${ }^{4}$ Department of Anesthesia, AHEPA University Hospital, Thessaloniki, Greece
}

\begin{abstract}
Rectus sheath hematoma (RSH) is an uncommon clinical event usually associated with trauma or coagulation disorders. It can also occur spontaneously. RSH usually runs a benign course but it can present with hypovolemic shock. When conservative management fails, surgical exploration becomes mandatory. We propose a technique of emergency ligation of the inferior epigastric artery. This is a simple approach as this artery originates at the posterior rectus sheath above the inguinal ligament. This safe technique provides immediate hemodynamic stabilization.
\end{abstract}

Keywords: Rectus sheath, hematoma, inferior epigastric artery.

\section{INTRODUCTION}

Rectus sheath hematoma (RSH) is an uncommon clinical event [1]. It can present spontaneously or in relation to trauma, coagulation disorders, coughing, physical exercise, obesity or pregnancy [2-5]. RSH is often initially misdiagnosed as it can mimic other acute abdominal conditions and present to a variety of specialists [6]. Usually RSH responds well to conservative management [3]. However, blood loss can be substantial leading to mandatory emergency surgery. In most cases the bleeding site is difficult to identify in the setting of an expanding hematoma. In such circumstances we propose immediate ligation of the inferior epigastric artery at its origin posterior to the rectus abdominis muscle as the procedure of choice. We illustrate this approach with a case report.

\section{CASE}

A 57 year old woman with ulcerative colitis on methotrexate and corticosteroids underwent total colectomy with formation of an ileoanal pouch. During the operation, there was an abrupt hemodynamic deterioration with a drop in hematocrit levels from 31 to $24 \%$ without any sign of intraperitoneal bleeding from the operative field. After the retractors were removed a large left RSH below the arcuate line was identified extending extraperitoneally.

An incision $1 \mathrm{~cm}$ above and parallel to the inguinal ligament at its medial third was made. The common femoral artery was dissected and the origin of the inferior epigastric

*Address correspondence to this author at the AHEPA University Hospital St. Kiriakidi 1, 54636 Thessaloniki Greece; Tel: 00302310994843;

Fax:00302310994871; E-mail: mantelas@gmail.com artery was identified. The inferior epigastric artery was ligated with an immediate hemodynamic improvement.

The patient had an uneventful recovery and was discharged from the hospital on the $9^{\text {th }}$ postoperative day with a minor bruise on the left lateral abdominal wall.

\section{DISCUSSION}

Teske in 1946 first presented 100 cases and observed that RSH is more frequent on the right side $(60 \%)$ and in the lower quadrants (80\%) [7]. This can be explained in part by the anatomy of the rectus sheath. The posterior sheath below the arcuate line consists only of a thin layer of transversalis fascia which separates the rectus abdominis muscle from the peritoneum. There is a female preponderance with a frequency of 2.5-3:1 [8] probably due to reduced muscle mass.

CT scan of the abdomen is the method of choice for the diagnosis of RSH [9]. Based on CT findings RSHs are divided into 3 types, according to severity [10]. In Type I, the bleeding is intramuscular with minimal hemodynamic compromise, in Type II there is blood between the muscle and the transversalis fascia and in the more severe Type III there is a marked hematoma that extends to the prevescical space and sometimes intraperitoneally. Our case can be characterized as Type III due to the extraperitoneal extension that led to a drop in hematocrit and hypovolemic shock. Abdominal ultrasound has also been used to diagnose RSH [5].

Treatment is conservative and symptomatic in most hemodynamically stable cases of RSH $[9,11]$. Surgical management is usually performed in an emergency setting in patients with hypovolemic shock $[9,11]$. Surgery consists of exploration of the rectus sheath often with few findings. Lack of control of bleeding is associated with high mortality [2]. 
Coil embolisation of the bleeding vessels can be considered as an alternative in high-risk cases. However, this technique is time-consuming, expensive and not always available. This procedure is also associated with complications such as contrast-induced nephropathy [12] or bleeding from the puncture site [13]. Another disadvantage is that the bleeding vessel cannot always be identified.

Considering the fact that the blood supply of the lower part of the rectus muscle is derived almost exclusively from the inferior epigastric artery, if this is interrupted proximally the bleeding will cease. In our case, proximal ligation of the inferior epigastric artery led to immediate hemodynamic stabilization and resolution of the expanding hematoma. There was no local recurrence on follow-up. In extreme situations inferior epigastric vessel ligation can be performed under local anesthesia at the bedside providing time for hemodynamic improvement and transfer of the patient to the operating theatre. Further evidence is required in order to establish the efficacy of this method. Considering that this technique is safe, rapid and relatively easy to perform, even by relatively inexperienced surgeons, we suggest this to be the first choice before surgical exploration for a severely bleeding RSH.

\section{REFERENCES}

[1] Dubinsky IL. Hematoma of the rectus abdominis muscle: case report and review of the literature. J Emerg Med 1997; 15: 165-7.
[2] Kapan S, Turhan AN, Alis H, et al. Rectus sheath hematoma: three case reports. J Med Case Rep 2008; 2: 22.

[3] Linhares MM, Lopes Filho GJ, Bruna PC, Ricca AB, Sato NY, Sacalabrini M. Spontaneous hematoma of the rectus abdominis sheath: a review of 177 cases with report of 7 personal cases. Int Surg 1999; 84: 251-7.

[4] Lambroza A, Tighe MK, DeCosse JJ, Dannenberg AJ. Disorders of the rectus abdominis muscle and sheath: a 22-year experience. Am J Gastroenterol 1995; 90: 1313-7.

[5] Klingler PJ, Wetscher G, Glaser K, Tschmelitsch J, Schmid T, Hinder RA. The use of ultrasound to differentiate rectus sheath hematoma from other acute abdominal disorders. Surg Endosc 1999; 13: 1129-34.

[6] Luhmann A, Williams EV. Rectus sheath hematoma: A series of unfortunate events. World J Surg 2006; 30: 2050-5.

[7] Teske JM. Hematoma of the rectus abdominis muscle: report of a case and analysis of 100 cases from the literature. Am J Surg 1946; 71: 689-95.

[8] Cherry WB, Mueller PS. Rectus Sheath Hematoma: review of 126 cases at a single institution. Medicine (Baltimore) 2006; 85: 105-10.

[9] Salemis NS, Gourgiotis S, Karalis G. Diagnostic evaluation and management of patients with rectus sheath hematoma. A retrospective study. Int J Surg 2010; 8: 290-3.

[10] Berna JD, Garcia-Medina V, Guirao J, Garcia-Medina J. Rectus sheath hematoma: diagnostic classification by CT. Abdom Imaging 1996; 21: 62-4.

[11] Fitzgerald JE, Fitzgerald LA, Anderson FE, Acheson AG. The changing nature of rectus sheath haematoma: case series and literature review. Int J Surg 2009; 7 : 150-4.

[12] Paraskevas KI, Giannoukas AD, Kotsikoris I, Mikhailidis DP Contrast-Induced Nephropathy and the Vascular Patient. Angiology 2010 Aug 3. [Epub ahead of Print]

[13] Zack JR, Ferral H, Postoak D, Wholey M. Coil embolization of rectus sheath hemorrhage. J Trauma 2001; 51: 793-5. 